\title{
RECOMENDAÇÕES PARA SE EVITAR GRANDES ERROS DE DOSE EM TRATAMENTOS RADIOTERAPÊUTICOS
}

\author{
Cleber Nogueira de Souza ${ }^{1}$, Carlos Roberto Monti ${ }^{2}$, Cláudio Hissao Sibata ${ }^{3}$
}

Resumo Erros humanos são uma importante fonte de falhas em todos os passos do planejamento e do tratamento radioterapêutico. Com o objetivo de reduzir este grau de incerteza, várias organizações especializadas recomendam minuciosos programas de garantia da qualidade. No Brasil, programas deste tipo vêm tendo sua exigência intensificada, e a maioria dos serviços de radioterapia vem se orientando neste sentido, tanto em relação aos equipamentos de radiação e dosimetria, quanto em relação à verificação dos cálculos de dose em pacientes e das revisões das fichas de planejamento. Como uma contribuição a este esforço de qualidade, apresentam-se algumas recomendações para se evitar falhas de tratamento devidas a erros na dose de radiação recebida pelo paciente, como redundância nas verificações dos cálculos feitos manualmente ou por computador, e, também, a verificação da dose acumulada para cada paciente sob tratamento, semanalmente, além de se evitar a possibilidade de acesso a qualquer sistema de segurança do equipamento ao pessoal técnico treinado para apenas o operar. Além disso, deve-se considerar a possibilidade de se empregar um sistema computadorizado de verificação e registro do tratamento, dessa maneira prevenindose erros durante a aplicação diária devidos à seleção indevida dos diferentes parâmetros do tratamento. Reportam-se quatro incidentes radioativos recentes ocorridos no mundo, com injúrias em pacientes, e algumas ocorrências de erros grandes de dose.

Unitermos: Radioterapia. Tratamento. Erros de dose. Garantia da qualidade.

Abstract Recommendations to avoid gross errors of dose in radiotherapeutic treatments.

Human mistakes are an important source of errors in radiotherapy and may occur at every step of the radiotherapeutic planning and treatment. To reduce this level of uncertainties, several specialized organizations have recommended a comprehensive quality assurance program. In Brazil, the requirement for these programs has been strongly stressed, and most radiotherapy services have pursued this goal regarding radiation units and dosimetry equipment, as well as the verification of the calculations of the patient's dose and the revision of the plan charts. As a contribution to the improvement of quality control, we present some recommendations to avoid failure of treatment due to error in the delivered dose, such as redundant check of the manual or computer calculations, weekly check of the total dose for each patient, and prevention of inadvertent access to any safety system of the equipment by any staff member that is only supposed to operate the machine. Moreover, the use of a computerized treatment record and verification system should be considered in order to eliminate errors due to incorrect selection of the treatment parameters, in a daily basis. We report four radioactive incidents with patient injuries occurred throughout the world and some gross errors of dose.

Key words: Radiotherapy. Treatment. Dose errors. Quality assurance.

\section{INTRODUÇÃO}

O sucesso ou a falha de um tratamento por radiação depende da dose liberada no volume inteiro do tumor, e não deve variar mais que $5 \%$ da dose prescrita $^{(\mathbf{1})}$. Esta técnica terapêutica consiste em um conjunto de procedimentos que

1. Físico, D.Sc., Instituto de Pesquisas Energéticas e Nucleares, São Paulo, SP.

2. Médico, D.Sc., Instituto do Radium de Campinas, Campinas, SP.

3. Físico, Ph.D., Case Western Reserve University, Cleveland, EUA

Endereço para correspondência: Dr. Cleber Nogueira de Souza. Instituto de Pesquisas Energéticas e Nucleares. Travessa R, 400, Cidade Universitária. São Paulo, SP, 05508-900.

Aceito para publicação em 4/12/2000. pode ser dividido da seguinte maneira: a) a prescrição do tratamento, que é uma decisão médica; b) a preparação para tratamento, que inclui a aquisição de dados do paciente, radiografias, definição de volume alvo e órgãos críticos, a simulação do tratamento, o cálculo da distribuição da dose, o cálculo da dose monitor ou do tempo de irradiação, e a preparação da ficha de tratamento; c) a execução do tratamento, que consiste na imobilização e posicionamento diários do paciente, a seleção dos parâmetros de tratamento e a irradiação, que pode incluir verificações de filmes "portals", o uso de sistemas de visão "portal" computadorizada e dosimetria in vivo.
Cada um desses procedimentos está sujeito a um certo grau de incerteza relacionado a falhas humanas ${ }^{(2-9)}$, que resultam, de maneira geral, em pequenos erros. Estas falhas, no entanto, podem também causar sérios acidentes ${ }^{(\mathbf{1 0}-16)}$.

Com o objetivo de reduzir este grau de incerteza, várias organizações especializadas recomendam minuciosos programas de garantia da qualidade ${ }^{(1,17,18)}$. No Brasil, programas deste tipo vêm tendo sua exigência intensificada ${ }^{(\mathbf{1 9 , 2 0 )}}$, e a maioria dos serviços de radioterapia vem-se orientando neste sentido, tanto em relação aos equipamentos de radiação e dosimetria, quanto em relação à verificação dos cálculos de dose em pa- 
cientes e da qualidade das revisões das fichas de planejamento.

Como uma contribuição a este esforço de qualidade, apresentamos algumas recomendações para se evitar falhas de tratamento devidas a erros na dose de radiação prescrita. Além disso, são reportados alguns incidentes radioativos recentes e alguns casos da ocorrência de tais erros. Pretende-se, neste artigo, reforçar aos profissionais da área de radioterapia a importância e a necessidade de programas de garantia da qualidade em radioterapia que se estendam aos próprios cálculos dos planejamentos, sejam eles feitos manualmente ou via computador, além do controle da qualidade dos equipamentos emissores de radiação. Estas observações são originadas da experiência acumulada por diferentes centros internacionais nos procedimentos de controle da qualidade de tratamentos radioterapêuticos ${ }^{(2,21-25)}$.

\section{REGISTROS RECENTES DE GRANDES ERROS DE DOSES EM PACIENTES}

Alguns incidentes radioativos, envolvendo grandes erros de dose em pacientes, têm sido reportados na literatura, devido a negligência ou falha do programa de garantia da qualidade. Entre 1974 e 1976, no Riverside Methodist Hospital, Columbus, EUA, 426 pacientes receberam sobredose de radiação em tratamento por cobaltoterapia, o que acarretou alta incidência de efeitos tardios em tecidos normais ${ }^{(12)}$. $\mathrm{O}$ fato se deveu à calibração inapropriada da unidade de cobalto por causa de um erro na correção do decaimento da fonte. Isto resultou em um efeito cumulativo, com o aumento do erro com o tempo, que alcançou sobredose de 50\% nos pacientes, quando foi descoberto.

Entre 10 e 20 de dezembro de 1990, no Hospital Clínico Universitário, Zaragoza, Espanha, 27 pacientes receberam sobredose de radiação em tratamento sob feixes de elétrons de um acelerador linear ${ }^{(11)}$. O acelerador apresentou um defeito de operação, que levou à irradiação dos pacientes com a energia de elétrons disponível mais alta, de $36 \mathrm{MeV}$, mesmo quando a energia selecionada era muito mais baixa. A dose de radiação desses pacientes foi de até sete vezes a dose pretendida no volume alvo, assim como a dose em profundidade em tecidos sadios. Na Europa, entre 1983 e 1991, foram registrados 1.344 casos de injúrias sérias em pacientes, conseqüentes a incidentes radioativos similares ${ }^{(14)}$.

Em setembro de 1996, em São José da Costa Rica, 109 pacientes foram sobre-irradiados, devido a um erro na calibração de uma unidade de cobaltoterapia, após a troca da fonte. O erro só foi detectado em outubro de 1996, quando vários pacientes apresentaram graves sintomas de sobre-exposição. O principal erro de calibração encontrado ocorreu quando um tempo de exposição definido de 30 segundos foi utilizado em lugar de 0,3 minuto. $O$ fator de extradose foi de 1,72 . Depois da avaliação da unidade e ajuste do indicador de distância de tratamento, que estava fora de especificação, a unidade foi recalibrada. $\mathrm{Na}$ avaliação das doses tumores, os erros encontrados foram de 1,32 a 1,84 vez a dose pretendida. Estas diferenças foram atribuídas a uma falha no programa de cálculo de dose computadorizado.

Em novembro de 1996, no Brasil, ocorreu um incidente envolvendo oito pacientes sob tratamento em feixes de elétrons de 6 a $12 \mathrm{MeV}^{(15)}$. Neste caso, uma falha de operação foi sobrepassada por intermédio da mudança de operação da máquina do modo normal (modo clínico) para o modo pesquisa ("research"), motivada por um defeito intermitente de planura/simetria ("flat/sym") apresentado pelo equipamento. Ocorreu, porém, que a chave "pgm/norm", que define os parâmetros do feixe programado, foi, inadvertidamente, acionada para a posição "prgm". Isto provocou uma dose de cerca de oito vezes o valor da dose pretendida ${ }^{(16)}$.

\section{PROCEDIMENTOS PARA SE EVITAR GRANDES ERROS DE DOSE EM PLANEJAMENTO}

Consideram-se grandes erros de dose na irradiação de pacientes sob tratamento radioterapêutico aqueles que resul- tem diferença na dose pretendida superior a $10 \%{ }^{(21)}$. Estes erros podem ser de vários tipos, como, por exemplo, erros de cálculos aritméticos, utilização errada nos dados de máquina, falha de interpretação das instruções escritas, uso incorreto do fator de decaimento de fonte radioativa, uso de fatores inadequados em cálculo, sobreposição de campos de tratamento e falha de funcionamento da unidade de tratamento.

As seguintes sugestões podem contribuir para que estes tipos de erros sejam evitados:

1. Considerar redundância uma virtude e não uma marca de ineficiência ${ }^{(24)}$. A redundância deve ser estendida a todos os procedimentos, teleterapia e/ou braquiterapia, a cada paciente, devendo as verificações serem feitas por uma outra pessoa que não aquela que realizou os cálculos. Uma revisão independente de uma pessoa de fora do grupo freqüentemente revela erros sistemáticos.

2. Não confiar, totalmente, em cálculos feitos por computador ${ }^{(23,26)}$. É boa prática checar todo plano de tratamento computadorizado por alguma verificação manual, por exemplo, de um ponto único, fácil de ser calculado. A saída estilizada de dados do sistema de planejamento computadorizado, às vezes, confere credibilidade indevida ao sistema.

3. Verificar a dose acumulada para os pacientes, semanalmente. Literalmente, centenas de erros e inconsistências têm sido descobertos durante a revisão das fichas de planejamento ${ }^{(21)}$.

4. Não permitir ao pessoal técnico, que opera o equipamento de irradiação, acesso aos seus sistemas de segurança ou de intertravamento ${ }^{(\mathbf{1 5})}$.

Algumas falhas nas próprias fichas de planejamento podem levar a erros de tratamento, como, por exemplo:

1. Prescrição do radioterapeuta: a) omissão de algum parâmetro de tratamento; b) letra manuscrita ilegível; c) falha na escrita de mudança da prescrição de tratamento; d) falha na posição do tumor (lado esquerdo/lado direito).

2. Dosimetrista ou técnico: a) cálculo incorreto de tempo; b) erros de cálculo da dose diária máxima e dose tumor; c) registro incorreto da posição exata do 
paciente; d) medidas incorretas das dimensões do paciente; e) erros ao se fazer mudanças em cálculos baseados em mudanças de parâmetros de tratamento ou do rendimento da máquina; f) erro dos parâmetros dosimétricos dos acessórios.

3. Técnico: a) erros de adição; b) erro na seleção do tempo de irradiação ou da dose monitor; c) erros nos parâmetros de posição do paciente; d) falhas na observação de mudanças nos parâmetros do tratamento; e) falhas na observação de mudanças na prescrição; f) falhas no emprego de acessórios prescritos para o tratamento.

Algumas recomendações, mais gerais, poderiam ser adotadas: identificar áreas problemáticas, fazer uma análise da sequiência dos eventos. Por exemplo, verificar se a mesma pessoa acompanha o paciente durante a simulação e o planejamento. Às vezes, uma pessoa inicia o trabalho no paciente e esquece de transmitir, verbalmente ou por escrito, informações sobre o paciente. Quando houver dúvidas básicas, por exemplo, se o lado do tratamento é o esquerdo ou o direito, encaminhar-se direto à fonte de informação: a prescrição do médico ou o exame histopatológico.

Deve-se considerar que errar é humano. A crença em erro humano nulo é utópica e uma atitude suicida. A questão mais problemática é o que fazer quando um erro é descoberto, embora tenha se passado por uma verificação. Nesta situação, o melhor é analisar todo o processo radioterapêutico, em vez de colocar mais uma verificação por outro profissional, alterando o processo onde necessário, a fim de se eliminar o erro. Um exemplo de situação deste tipo poderia ser aquela em que várias bandejas são utilizadas. Neste caso, poder-se-ia eliminar algumas bandejas, ou estabelecer um fator de bandeja médio, permitindo uma variação na dose calculada igual à diferença entre a média dos fatores bandeja e a bandeja utilizada.

$\mathrm{O}$ risco de erro com o uso de computadores tornar-se catastrófico existe; no entanto, eles são melhores do que os seres humanos para a verificação de dados, pois não apresentam cansaço ou té- dio. Neste caso, sempre deve-se verificar as informações colocadas no sistema computacional, e ter a certeza absoluta do resultado fornecido pelo sistema computacional.

Um sistema computacional para a minimização de erros a ser considerado em um serviço de radioterapia é aquele denominado de "sistema de registro e verificação" (SRV - "record and verify system")(27). Este tipo de sistema melhora a precisão dos tratamentos liberados, pois evita que um tratamento seja executado, a menos que todas as informações originais para o tratamento, como ângulo do "gantry", ângulo do colimador, tamanho do campo, etc., combinem com as informações da aplicação em curso. Entretanto, excessiva confiança em um SRV pode levar a erros repetidos, pois existe um potencial para a entrada de parâmetros errados no sistema, de forma que estes erros se tornem sistemáticos. Por isso, toda informação inserida em um SRV deve ser independentemente verificada.

\section{DESCRIÇÃO DE ALGUMAS SITUAÇÕES REPORTADAS DE ERROS MAIORES QUE 10\% NA DOSE PRESCRITA ${ }^{(21)}$}

\section{Falhas envolvendo o uso de equipamentos de teleterapia}

Falha $n^{\circ} 1$

Causa: Erro na medida do fator de transmissão de um filtro em cunha.

Fator que contribuiu para a falha: a) Nenhuma verificação independente das medidas de transmissão; b) falha do pessoal técnico em comparar com medidas prévias a tempo.

Quando o erro foi detectado: Três anos após o uso do parâmetro incorreto.

Como o erro foi detectado: Durante a calibração completa da unidade de cobaltoterapia, para implementação de um novo sistema de planejamento computadorizado, todas as medidas dos modificadores de feixe foram refeitas.

Principal pessoa credenciada envolvida: Físico.

Ações corretivas propostas: Requerer verificação independente dos parâmetros de medida.
Descrição: O erro foi cometido por ocasião da medida dos fatores de filtro em cunha. Este erro resultou em tratamento com dose menor que a prescrita de pelo menos 53 pacientes de um total de 900, tratados no período de julho/ 1980 a agosto/1983. A maioria dos pacientes recebeu dose de $10 \%$ a $15 \%$ menor que a dose prescrita. Foi possível contatar 38 pacientes, e em três casos havia ocorrido recidivas da neoplasia.

$\mathrm{O}$ erro foi detectado durante as medidas anuais de rendimento do equipamento, que incluem a verificação de todos os fatores dos filtros utilizados no tratamento de pacientes. Assim, por exemplo, o filtro em cunha de ângulo nominal de $60^{\circ}$, que tinha fator de uso de 1,63 , no período do acontecimento, passou a ter novo fator, igual a 2,70 .

\section{Falha $n^{\circ} 2$}

Causa: Erro humano na localização do campo de tratamento.

Fator contribuinte: Nenhuma verificação independente da localização do tratamento.

Quando o erro foi detectado: Depois de uma aplicação.

Como o erro foi detectado: Revisão do plano de tratamento.

Principal pessoa credenciada envolvida: Técnico.

Ações corretivas propostas: Uma segunda verificação independente do tratamento.

Descrição: Um paciente foi simulado para tratamento da mama direita, em uma unidade de cobalto-60, quando a mama esquerda é a que deveria ser irradiada. O paciente recebeu uma aplicação de 200 cGy e não apresentou nenhuma seqüela aparente.

\section{Falha $n^{\circ} 3$}

Causa: Erro de multiplicação no cálculo do tempo de exposição em uma unidade de cobalto-60.

Fator contribuinte: a) Nenhuma verificação independente; b) falha da pessoa credenciada em fazer dupla verificação dos cálculos em tempo hábil.

Quando o erro foi detectado: Dois meses após o final do tratamento.

Como o erro foi detectado: Revisão da ficha de planejamento. 
Principal pessoa credenciada envolvida: Dosimetrista.

Ações corretivas propostas: Requerer que todos os cálculos de tempo de exposição sejam verificados por uma segunda pessoa.

Descrição: Um paciente, agendado para tratamento em uma unidade de cobalto-60 para dose total de 3.000 cGy, recebeu 3.345 cGy $(11,5 \%$ a mais da dose prescrita). O período de tratamento foi de 22 de dezembro a 6 de janeiro do ano seguinte (dez seções). O erro foi descoberto em 10 de março. A irradiação extra ocorreu devido a um erro no cálculo do tempo de exposição da fonte.

\section{Falha $n^{0} 4$}

Causa: O técnico cometeu erro de leitura do tempo de exposição calculado; em vez de se ler 1,39 minuto, leu-se 1,89 minuto.

Fator contribuinte: A pessoa credenciada falhou na realização da revisão da ficha de planejamento.

Quando o erro foi detectado: Depois da $11^{\mathrm{a}}$ aplicação das 12 prescritas.

Como o erro foi detectado: Revisão da ficha de planejamento do paciente.

Principais pessoas credenciadas envolvidas: Técnico, dosimetrista, físico.

Descrição: Um tratamento de 3.000 cGy para a linha média do cérebro em 12 frações iguais de $250 \mathrm{cGy}$, por intermédio de dois campos laterais, foi prescrito. O tempo de tratamento calculado para cada feixe foi de 1,39 minuto.

O dosimetrista calculou o tempo de tratamento na folha de cálculo da física e então assinalou, com lápis, na primeira coluna da folha de registro de tratamento. Os cálculos foram então checados por um físico, que rubricou, tanto a folha de cálculo quanto a coluna da folha de registro de tratamento.

De acordo com os procedimentos do hospital, após realizada a primeira aplicação, o tempo de tratamento e as doses que tenham sido escritas a lápis pelo dosimetrista são confirmadas com caneta em cima da escrita a lápis pelo técnico que administra o tratamento.

Neste caso, o primeiro tratamento foi administrado por 1,89 minuto, em vez de 1,39 minuto. Este erro foi então co- metido durante mais dez seções de tratamento. $\mathrm{O}$ erro só foi descoberto após a $11^{\mathrm{a}}$ seção de tratamento. A $12^{\mathrm{a}}$ seção de tratamento foi cancelada. $\mathrm{O}$ paciente recebeu 3.753 cGy, em vez da prescrição de $3.000 \mathrm{cGy}$.

\section{Falha $n^{\circ} 5$}

Causa: Erro de aritmética na adição das doses por seção de tratamento.

Fator contribuinte: Falha da verificação independente ou da conduta de revisão das fichas de tratamento.

Quando o erro foi detectado: Depois da finalização do tratamento.

Como o erro foi detectado: Revisão da ficha de tratamento.

Principais pessoas credenciadas envolvidas: Técnico, dosimetrista, físico.

Ações corretivas propostas: Organizar novo comitê para revisão de nova ficha de planejamento; mudança do formato de registro para melhorar o sistema de verificação cruzada.

Descrição: Um paciente com metástase de carcinoma de mama reclamou, junto ao médico, de inchaço no braço esquerdo, devido a extensão da doença na axila esquerda. $\mathrm{O}$ tratamento da axila esquerda com radiação gama do cobalto60 iniciou-se em 2 de março. O tratamento finalizou-se em 20 de abril, depois de uma dose total de $6.173 \mathrm{cGy}$.

A falha de tratamento envolveu o registro da dose de irradiação. Inicialmente, o tratamento prescrito foi revisto diariamente, mas a rápida resposta do tumor levou a um regime de tratamento de três semanas para uma dose total de 4.575 cGy. O resultado, ao final de três semanas, foi tão favorável que um tratamento complementar foi prescrito pelo radioterapeuta. O tratamento foi interrompido pelo radioterapeuta após registro de dose tumor de 5.373 cGy, com o paciente em boas condições. Mais tarde, foi determinado que teria havido um erro de aritmética na adição das seções de tratamento e que a dose tumor real liberada era de 6.173 cGy para a axila.

Este erro foi encontrado pela divisão de física durante a verificação de rotina dos registros, quando a ficha de registro estava sendo retirada para liberação do paciente.

\section{Falha $n^{\circ} 6$}

Causa: Erro na determinação da profundidade do tumor. A profundidade definida, de $16,5 \mathrm{~cm}$, na ficha do paciente, era, de fato, de $11,5 \mathrm{~cm}$.

Fator contribuinte: a) Falha em verificar a profundidade do tumor antes de se iniciar o tratamento do paciente; b) falha na finalização da verificação de controle de qualidade de todos os fatores que determinam a dose no paciente durante o período de tratamento.

Quando o erro foi detectado: Durante a visita de seguimento do paciente, após o término do tratamento.

Como o erro foi detectado: Nova medida da profundidade do tumor pelo radioterapeuta.

Principal pessoa credenciada envolvida: Dosimetrista.

Ações corretivas propostas: Tomar maior cuidado na determinação da profundidade do tumor e realizar uma segunda verificação independente do planejamento.

Descrição: Um paciente sob tratamento recebeu dose tumor de $5.570 \mathrm{cGy}$, em vez da dose tumor prescrita de 4.070 cGy. $\mathrm{O}$ erro ocorreu por causa de um erro na medida da profundidade do tumor. Antes do início do tratamento, a profundidade do tumor medida foi de $16,5 \mathrm{~cm}$ e o cálculo de dose se baseou neste valor. $\mathrm{Na}$ última das várias visitas de seguimento do paciente, o radioterapeuta mediu, novamente, o paciente, e descobriu que a profundidade do tumor deveria ter sido de $11,5 \mathrm{~cm}$.

\section{Falha $n^{0} 7$}

Causa: A profundidade de cálculo do tratamento foi assinalada errada na ficha do paciente.

Fator contribuinte: a) Nenhuma verificação independente dos parâmetros de tratamento; b) falha de verificação adequada ou em tempo hábil da ficha de tratamento.

Quando o erro foi detectado: Após finalizada a primeira fase do tratamento.

Como o erro foi detectado: Durante cálculos da segunda fase do tratamento.

Principais pessoas credenciadas envolvidas: Dosimetrista, físico. 
Ações corretivas propostas: Não foi proposta nenhuma ação corretiva.

Descrição: Um paciente, para o qual a dose prescrita era de $4.500 \mathrm{cGy}$, recebeu dose de 6.000 cGy. A falha ocorreu porque a profundidade de tratamento a ser aplicada para o cálculo foi incorretamente assinalada na ficha do paciente. Isto foi descoberto quando novos cálculos estavam sendo feitos para a segunda fase do tratamento.

\section{Falha $n^{\circ} 8$}

Causa: Técnico falhou em anotar, na folha de tratamento, mudança da requisição de redução na área de tratamento do tumor.

Fator contribuinte: Nenhum.

Quando o erro foi detectado: Ao final do tratamento.

Como o erro foi detectado: Revisão da ficha de planejamento do paciente.

Principal pessoa credenciada envolvida: Técnico.

Ações corretivas propostas: Registrar todos os procedimentos do tratamento na ficha de prescrição.

Descrição: Um erro resultou na irradiação da área pulmonar de 2.475 cGy, em vez da prescrição de 1.500 a 1.800 cGy. A prescrição original foi de 3.000 cGy em toda a área inicial da doença no pulmão. Como o radioterapeuta percebeu que o volume de pulmão envolvido poderia não suportar tal dose, ele decidiu reduzir a área do tumor irradiada após 1.500-1.800 cGy de dose. Um bloco de proteção para reduzir a área que estava sendo irradiada não foi inserido como se pretendia, porque o próprio médico não tinha atualizado a prescrição, antes desta fase de tratamento.

\section{Falha $n^{\circ} 9$}

Causa: Dados errados foram gerados por um programa de computador e empregado em um planejamento, o que resultou em dose extra nas últimas três de um total de cinco aplicações.

Fator contribuinte: a) Falho controle de qualidade no uso de planejamento de tratamento computadorizado; b) falha na verificação dos resultados dos dados de tratamento gerados.

Quando o erro foi detectado: Depois do final do tratamento.
Como o erro foi detectado: Revisão da ficha de planejamento.

Principais pessoas credenciadas envolvidas: Dosimetrista, físico.

Ações corretivas propostas: a) Remover os dados errados do computador; b) verificar os futuros planos de tratamento por computador por intermédio de cálculo manual em um ponto no eixo central; c) comparar todas as curvas de isodose dos feixes externos com dados medidos, antes de empregar o sistema de planejamento computadorizado.

Descrição: Um paciente sob teleterapia do lado direito da região torácica posterior foi irradiado com a dose de 3.200 cGy, em vez da dose prescrita de $2.000 \mathrm{cGy}$. O tratamento foi realizado em duas aplicações de 400 cGy e três aplicações de 800 cGy.

A falha ocorreu, aparentemente, devido à combinação de dados errados do computador do sistema de planejamento e o respectivo cálculo baseado nestes dados. O erro foi descoberto quando os cálculos foram novamente verificados, depois do final do tratamento.

\section{Falha $n^{\circ} 10$}

Causa: Parâmetros de tratamento calculados manualmente foram empregados para duas aplicações, e então a análise dos dados foi feita por intermédio de um computador. Desta análise, um erro foi feito na conversão de cGy por dia para tempo de exposição.

Fator contribuinte: a) Nenhuma verificação independente dos cálculos de dose do paciente; b) falha na realização de verificação adequada ou em tempo hábil da ficha de planejamento.

Quando o erro foi detectado: Depois da finalização do tratamento.

Como o erro foi detectado: Revisão da ficha de planejamento.

Principais pessoas credenciadas envolvidas: Dosimetrista, físico.

Ações corretivas propostas: Garantir a realização de verificações cruzadas do planejamento em tempo hábil.

Descrição: Um paciente programado para receber $3.000 \mathrm{cGy}$ em um tratamento de cobaltoterapia recebeu, em vez disto, 3.614 cGy. O tratamento prescrito foi de uma dose tumor mínima total de 3.000
cGy para o cérebro, a ser liberada em dez frações, por intermédio de dois campos laterais paralelos e opostos, em uma unidade de cobalto-60. A falha foi detectada durante a verificação dosimétrica final, realizada pela divisão de física, que determinou que a dose total liberada no tumor foi de 3.614 cGy. Em 31 de julho, o cálculo manual foi feito corretamente para o tratamento do paciente, que receberia dose total de 300 cGy por dia dos dois campos laterais. Os parâmetros utilizados para este cálculo foram empregados em duas frações, a partir daí uma análise por computador foi feita para determinar um esquema de dose mais preciso para o resto do tratamento. Um erro na conversão de cGy/dia para minutos de exposição por dia ocorreu neste ponto do processo de planejamento, em 4 de agosto. Este tempo errado foi utilizado para as oito aplicações restantes.

\section{Falha $n^{0} 11$}

Causa: O dosimetrista utilizou um fator de taxa de dose da tabela de uma outra máquina.

Fator contribuinte: a) Nenhuma verificação independente dos cálculos de dose para o planejamento do paciente; b) fatores de taxa de dose para várias máquinas mantidas na mesma pasta.

Quando o erro foi detectado: Depois de 27 aplicações.

Como o erro foi detectado: O dosimetrista detectou o erro enquanto revisava o plano do tratamento.

Principais pessoas credenciadas envolvidas: Dosimetrista, físico.

Ações corretivas propostas: a) Separar os dados de cada máquina em diferentes pastas; b) entrar com o fator de taxa de dose usado nos cálculos dosimétricos no plano de tratamento do paciente.

Descrição: Um paciente sob tratamento em uma unidade de cobaltoterapia recebeu $8.100 \mathrm{cGy}$, em vez da dose prescrita de $6.500 \mathrm{cGy}$. O erro no tratamento foi detectado pelo dosimetrista, durante revisão do tempo de tratamento do paciente para um outro procedimento. Na época em que o erro foi detectado, o registro de tratamento do paciente indicava que havia sido liberada dose 
acumulada de 4.860 cGy em 27 frações de $180 \mathrm{cGy} / \mathrm{dia}$, embora o paciente tivesse recebido, de fato, $8.100 \mathrm{cGy}$ em frações diárias de 300 cGy, durante as 27 aplicações.

O plano de tratamento para este paciente foi realizado em um determinado hospital, e então o paciente foi transferido para um outro hospital, onde o tratamento foi realizado. Para o cálculo do tempo de tratamento foi necessário que o dosimetrista ou o físico obtivesse a taxa de dose calibrada para uma máquina determinada, a partir de uma tabela de taxa de dose versus tamanho de campo. Os dados referidos para três máquinas diferentes eram mantidos em uma mesma pasta. $\mathrm{O}$ erro ocorreu porque $\mathrm{o}$ dosimetrista obteve a taxa de dose para uma máquina diferente daquela que deveria ser utilizada, e o físico que revisou o planejamento não detectou o erro.

Falha $n^{\circ} 12$

Causa: O dosimetrista cometeu um erro ao calcular o tempo de exposição que seria utilizado para uma dose diária de 200 cGy por campo. Este valor, no entanto, foi reduzido para $150 \mathrm{cGy}$ por campo.

Fator contribuinte: a) Nenhuma verificação independente dos cálculos de dose do paciente; b) falha em realizar, em tempo hábil ou de forma adequada, verificação da ficha de planejamento.

Quando o erro foi detectado: Após 24 aplicações, sendo 15 frações de 400 cGy e nove frações de 300 cGy.

Como o erro foi detectado: Reação de pele do paciente levou a uma verificação dos tempos calculados.

Indicação do erro: Paciente desenvolveu eritema, que se intensificou com o tratamento.

Principais pessoas credenciadas envolvidas: Médico, dosimetrista, físico.

Ações corretivas propostas: Uma segunda verificação pelo físico dos cálculos dosimétricos, antes da terceira fração de tratamento.

Descrição: Um paciente recebeu dose no cérebro de $8.700 \mathrm{cGy}$, em vez da dose prescrita de $6.000 \mathrm{cGy}$. O tratamento prescrito para o paciente foi de $200 \mathrm{cGy}$ de dose por dia para a linha média do cérebro, por intermédio de dois campos laterais paralelos e opostos. Dessa maneira, cada campo lateral deveria contribuir com 100 cGy/dia para esta dose diária prescrita. O paciente recebeu taxa de dose de 400 cGy/dia (200 cGy/dia por campo).

O paciente desenvolveu eritema durante o tratamento. Uma vez que esta reação foi mais severa do que o previsto, o médico reduziu a dose total por dia para 150 cGy a partir da $15^{\mathrm{a}}$ aplicação. Um segundo dosimetrista calculou o novo tempo de irradiação, repetindo o erro original. O dosimetrista que calculou a dose de tratamento inicial errou ao calcular um tempo de irradiação para dar 200 cGy de cada lado do crânio por dia (duas vezes a quantidade de radiação prescrita).

A intensidade do eritema continuou a aumentar, e depois de nove aplicações com a dose diária reduzida o médico solicitou revisão dos cálculos. A verificação dos cálculos identificou o erro e o tratamento foi interrompido. O paciente tinha recebido $8.700 \mathrm{cGy}$, isto é, $45 \%$ a mais da dose prescrita.

\section{Falha $n^{0} 13$}

Causa: Dosimetrista e físico interpretaram erroneamente a prescrição médica e calcularam o tempo de máquina para uma dose de 200 cGy por campo de tratamento por fração, em vez de 100 cGy por campo de tratamento.

Fator contribuinte: a) Nenhuma verificação independente dos cálculos de dose do paciente; b) verificação inadequada das fichas de planejamento dos pacientes.

Quando o erro foi detectado: Depois de 16 aplicações.

Como o erro foi detectado: Durante a revisão de rotina das fichas dos pacientes.

Principais pessoas credenciadas envolvidas: Médico, dosimetrista, físico.

Ações corretivas propostas: a) Restabelecer procedimentos próprios para o cálculo e a especificação da dose tumor para técnicas de tratamento com múltiplos campos; b) reenfatizar a importância da revisão das fichas dos pacientes; c) haver mais de uma verificação individual da prescrição de dose. Se a prescrição for ambígua, esta ambigüidade deve ser resolvida com o médico.

Descrição: Um paciente em teleterapia para tratamento do cérebro, com dose prescrita de $4.000 \mathrm{cGy}$, recebeu dose de 6.400 cGy na área de tratamento. A dose foi administrada em um campo frontal e outro lateral da cabeça (100 cGy de cada lado). Calculou-se, erroneamente, o tempo de exposição, de tal maneira que o paciente recebeu 200 cGy no campo frontal e 200 cGy no campo lateral. O tempo de exposição total foi, assim, o dobro do prescrito para cada fração.

O paciente recebeu 16 frações em nível elevado de dose, totalizando 6.400 cGy, comparada com a dose prescrita de $4.000 \mathrm{cGy}$. O erro foi descoberto durante revisão dos registros do tratamento pelo médico.

Embora houvesse procedimento de controle da qualidade para verificar os cálculos de tempo de exposição, este, aparentemente, falhou em detectar o erro em tempo.

\section{Falha $n^{\circ} 14$}

Causa: Inicialmente, o médico prescreveu, verbalmente, dose de 600 cGy a ser dada em três frações. Ele, mais tarde, assinou prescrição de 300 cGy a ser dada em cinco frações. A confusão com as duas prescrições resultou em plano de tratamento de 600 cGy em cinco frações. O físico, que tinha verificado os cálculos de dose para a prescrição fornecida verbalmente, transferiu os dados deste cálculo para a folha de tratamento.

Fator contribuinte: a) Emprego, inicial, de prescrição verbal, com a finalidade de colocar o paciente em simulação pelo técnico; b) verificação inadequada das fichas de planejamento.

Quando o erro foi detectado: Depois do tratamento finalizado.

Como o erro foi detectado: Revisão da ficha de planejamento, após o paciente ter reclamado de desconforto.

Indicação de erro: Um técnico perguntou ao físico a respeito do longo tempo para um tratamento.

Principais pessoas credenciadas envolvidas: Médico, dosimetrista, físico.

Ações corretivas propostas: a) Uso de prescrição escrita para realizar os cálcu- 
los de dose; b) antes da primeira fração, requerer verificação dos cálculos de dose pelo técnico e pelo físico, e verificação do número total de campos e da dose tumor por campo.

\section{Falha $n^{\circ} 15$}

Causa: Erro de cálculo pelo dosimetrista (verificado pelo físico, sem, no entanto, detectar o erro), que resultou em 154 cGy para cada um dos dois campos de tratamento, em vez de 100 cGy para cada campo, conforme prescrito.

Fator contribuinte: a) Revisão inadequada dos cálculos de dose pelo físico; b) revisão inadequada da ficha durante o transcorrer do tratamento.

Quando o erro foi detectado: Depois de 22 frações.

Como o erro foi detectado: O técnico responsável revisou o plano de tratamento, por causa das reações do paciente ao tratamento.

Principais pessoas credenciadas envolvidas: Médico, físico, técnico.

Indicação de erro: Severa reação do couro cabeludo.

Ações corretivas propostas: Requerer ao físico a realização de cálculos próprios, mais que a verificação dos cálculos feitos pelo técnico ou dosimetrista.

Descrição: Uma paciente com tratamento externo programado para ser realizado com cobalto-60 (irradiação de cérebro total) recebeu dose de $6.776 \mathrm{cGy}$, em vez da dose prescrita de 6.000 cGy. Os cálculos originais para irradiação com dois campos paralelos e opostos determinaram tempo de 1 minuto e 58 segundos para se liberar 100 cGy no plano médio sagital. Para este tempo de exposição, a dose de fato foi de 154 cGy no plano sagital médio para cada campo, resultando erro na dose diária de aproximadamente $50 \%$. A dosimetria original foi feita por um técnico na unidade de cobalto. O técnico fez o cálculo, que foi, subseqüentemente, verificado pelo físico residente. O físico revisou a dosimetria e os registros do tratamento em curso quatro vezes em um intervalo de duas semanas (procedimento usual no serviço). Depois de 22 frações e uma dose projetada de 4.000 cGy no plano sagital médio, o paciente apresentou reação for- te no couro cabeludo. Por causa disto, a dosimetria foi novamente verificada pelo físico, e nenhum erro encontrado. $\mathrm{O}$ tratamento foi interrompido. Três semanas mais tarde, quando a reação da paciente havia cedido, ela retornou ao tratamento; nesta ocasião, o técnico reviu o cálculo e observou um erro na dosimetria original. $\mathrm{O}$ tratamento tinha se iniciado em 22 de abril, o erro foi descoberto em 12 de junho.

\section{Falha $n^{\circ} 16$}

Causa: Funcionamento de emissor de fótons e elétrons no modo manual, por intermédio de mudança de posição de microchave junto ao console do aparelho, realizado pelo operador do equipamento, a fim de superar defeito de intertravamento, causado por aparente falha de planura e simetria do feixe.

Fator contribuinte: Próximo à chave de controle manual, existem outras chaves, que inclui a chave de programação dos parâmetros eletrônicos que determinam a energia e rendimento do feixe, que, inadvertidamente, teve sua posição alterada, permitindo a emissão de feixe de elétrons com parâmetros desconhecidos.

Quando o erro foi detectado: No mesmo dia em que ocorreu o defeito de falha e simetria dos feixes.

Como o erro foi detectado: Após corrigido o defeito, procedeu-se à dosimetria do equipamento em procedimentos de simulação dos tratamentos com as chaves nas posições alteradas.

Indicações de erro: Registro de falha de planura e simetria do feixe, grandes oscilações da taxa de dose, e indicativo luminoso de falha do feixe de elétrons.

Principais pessoas credenciadas envolvidas: Técnico, físico.

Ações corretivas propostas: $\mathrm{O}$ acesso ao sistema de chaves de controle de programação foi trancado a chave, de forma a ser acessível apenas ao físico responsável. Este acesso só será permitido em situações de testes do aparelho, sem a presença de paciente na sala de tratamento. Além da dosimetria e os testes diários e semanais, serão verificados, no início do dia, os fatores de calibração de todos os feixes, o que será repetido sem- pre que houver dúvida quanto ao funcionamento do aparelho. Todos os procedimentos, atribuições e responsabilidades relativos ao funcionamento do serviço de radioterapia serão revistos e rediscutidos. Será exigido maior rigor na assistência técnica do equipamento. Serão registrados, em livro próprio, pelos operadores do equipamento, todos os aspectos que se mostrarem fora de padrão, relativos ao equipamento e aos pacientes.

Descrição: O equipamento permite seu funcionamento nos modos manual e automático, por intermédio do acionamento de chave situada no console do aparelho. Este aparelho apresenta, às vezes, um falso bloqueio, que, na maioria dos casos, acusa falha na planura ou simetria do feixe ("FLAT/SYM"). Colocando o aparelho para funcionar no modo manual, o bloqueio fica desativado e o feixe é liberado. Como este defeito é freqüiente, foi, anteriormente, realizada dosimetria de todos os feixes nesta condição, e verificou-se que não havia alteração das características dos feixes de radiação nem no sistema de monitoração da dose que controla a interrupção automática do tratamento. Como este defeito é intermitente, as tentativas da assistência técnica para solucioná-lo, definitivamente, não foram bem sucedidas. Para que os tratamentos dos pacientes não fossem interrompidos devido a este defeito, que não altera a qualidade do tratamento, optou-se por trabalhar, quando necessário, no modo manual, até que o defeito fosse corrigido pelo técnico, o que, em geral, ocorre dentro de 24 horas. Este procedimento é comum e, muitas vezes, feito pelos próprios operadores do equipamento. No console do aparelho, junto ao local onde fica a chave que permite o seu funcionamento no modo manual, existem algumas microchaves que têm posições pré-estabelecidas, demarcadas no painel vizinho.

Num determinado dia, o aparelho acusou alguns bloqueios, que, após novas tentativas de funcionamento, desapareciam, e, de acordo com os operadores do aparelho, todos os pacientes foram tratados normalmente com a unidade no modo automático. 
No dia seguinte, pela manhã, o aparelho apresentou, várias vezes, problemas relacionados à "FLAT/SYM", quando operando com feixes de elétrons no modo automático. Por causa disto, até o meio da manhã, foram feitos os tratamentos dos pacientes submetidos ao feixe de fótons, apenas. Depois deste horário, com autorização do físico responsável, foram feitos os tratamentos dos pacientes submetidos a elétrons, no modo manual. Por volta do meio-dia, o equipamento apresentou grandes oscilações na taxa de dose, tendo sido constatado, pelo físico responsável, que a chave com a inscrição "PGM/NORM" estava na posição invertida, isto é, "PGM". Após a mudança da chave para a posição correta, "NORM", o aparelho voltou a funcionar com a taxa de dose usual, no modo automático. O aparelho continuou apresentando bloqueios intermitentes, e, em seguida, parou de funcionar, tendo sido observado que a lâmpada indicativa do funcionamento dos feixes de elétrons piscava.

\section{Falhas envolvendo o uso de equipamentos de braquiterapia}

Falha $n^{\circ} 17$

Causa: Fontes de atividades erradas foram carregadas no aplicador. O erro envolveu o carregamento de uma fonte de 17,4 mg rádio equivalente (codificada por um ponto alaranjado), em vez de uma fonte de $11,8 \mathrm{mg}$ rádio equivalente (codificada por um ponto branco).

Fator contribuinte: a) Nenhuma verificação independente da atividade da fonte pelo médico; b) as fontes não codificadas pela atividade, mas pelo código de cor; c) ambas as fontes guardadas na mesma gaveta.

Quando o erro foi detectado: Um dia após o final do tratamento (duração de 30 horas).

Como o erro foi detectado: Pelo físico, quando retornava as fontes ao cofre.

Indicações de erro: Os resultados do levantamento radiométrico da sala de tratamento, realizado após o carregamento das fontes, poderiam ter indicado nível de radiação maior que o esperado, para a atividade das fontes que estavam sendo utilizadas.
Principais pessoas credenciadas envolvidas: Dosimetrista (que carregou as fontes nos cateteres) e médico.

Ações corretivas propostas: a) Aconselhar aos dosimetristas requererem $\mathrm{o}$ inventório das fontes no momento da remoção e retorno delas; b) requerer que todo o pessoal do departamento de física que manuseie as fontes faça o inventório das fontes no cofre antes e depois da remoção ou retorno das fontes; c) segunda verificação do carregamento das fontes por uma pessoa independente.

Descrição: Um paciente sob tratamento externo e braquiterapêutico para câncer cervical recebeu doses $21 \%$ e $37 \%$ maiores que as doses prescritas ou planejadas, para as duas áreas de tratamento. A dose total da combinação da radiação externa e interna foi 3,5\% e $13 \%$ maior para as áreas afetadas do que as doses prescritas.

Este resultado ocorreu devido ao carregamento do aplicador com fontes com $17,4 \mathrm{mg}$ rádio equivalente, em vez de $11,8 \mathrm{mg}$ rádio equivalente.

\section{Falha $n^{\circ} 18$}

Causa: Fontes com atividades erradas foram carregadas em um aplicador de braquiterapia. O erro envolveu o carregamento de três fontes de $20,3 \mathrm{mg}$ rádio equivalente (código de cor branca), em vez de três fontes de $6,3 \mathrm{mg}$ rádio equivalente (código de cor púrpura).

Fatores contribuintes: a) Nenhuma verificação independente da atividade da fonte pelo médico; b) as fontes não estavam marcadas pela atividade, mas pelo código de cor; c) possível excessiva carga de trabalho.

Quando o erro foi detectado: Ao final do período de tratamento.

Como o erro foi detectado: Enquanto retornava as fontes ao cofre de armazenamento, o médico observou diferença entre o código de cores das fontes e a prescrição.

Indicações de erro: Os resultados do levantamento radiométrico da sala de tratamento, realizado após o carregamento das fontes, poderiam ter indicado nível de radiação maior que o esperado, para a atividade das fontes que estavam sendo utilizadas.
Principais pessoas credenciadas envolvidas: Técnico que carregava as fontes do cofre e o médico.

Ações corretivas propostas: a) Revisão do protocolo de manuseio das fontes; b) mudança da folha de registro das fontes, a fim de enfatizar a verificação do carregamento correto pelo médico, antes da aplicação.

Descrição: Paciente sob tratamento com césio-137 recebeu 12.000 cGy no útero, no período de 9 a 12 de abril. A dose total prescrita foi de 4.000 cGy. O erro ocorreu quando fontes de atividades erradas foram carregadas nos cilindros vaginais. O carregamento prescrito para o tratamento era de uma fonte com $9,9 \mathrm{mg}$ rádio equivalente e três fontes com $6,3 \mathrm{mg}$ rádio equivalente. $\mathrm{O}$ carregamento das fontes foi feito por um técnico registrado que estava fazendo treinamento como um dosimetrista. $\mathrm{O}$ erro foi descoberto pelo físico, durante o retorno das fontes ao cofre, após a remoção do paciente. $O$ físico observou que a codificação de cor da fonte não combinava com a cor da fonte prescrita (fontes de $20,3 \mathrm{mg}$ rádio equivalente são codificadas na cor branca, fontes de $6,3 \mathrm{mg}$ rádio equivalente são codificadas na cor púrpura). Um exame do paciente, três dias após a remoção das fontes, não revelou nenhuma anormalidade na vagina.

\section{CONCLUSÃO}

Existem várias situações que podem provocar grandes erros da dose na irradiação de pacientes sob radioterapia. Tais erros comprometem o sucesso do tratamento e devem ser minimizados ao máximo, mediante rígido controle da qualidade dos planejamentos de todos os pacientes. Alguns cuidados básicos podem ser tomados para que estas falhas não aconteçam, por exemplo, redundância nas verificações dos cálculos feitos manualmente ou por computador e, também, a verificação da dose acumulada para cada paciente sob tratamento, semanalmente, além de se evitar a possibilidade de acesso a qualquer sistema de segurança do equipamento ao pessoal técnico treinado para apenas o operar. Além disso, deve-se considerar a possi- 
bilidade de se empregar um sistema computadorizado de verificação e registro do tratamento; dessa maneira, previnem-se erros durante a aplicação diária devidos à seleção indevida dos diferentes parâmetros do tratamento.

\section{REFERÊNCIAS}

1. International Commission on Radiation Units and Measurements (ICRU). Determination of absorbed radiation in a patient irradiated by beams of $\mathrm{X}$ or gamma rays in radiotherapy procedures. Washington, DC: ICRU Report-24, 1976.

2. Valli MC, Prina M, Bossi A, et al. Evaluation of most frequent errors in daily compilation and use of a radiation treatment chart. Radiother Oncol 1994;32:87-9.

3. Dutreix A, van der Schueren E, Leunens L. Quality control at the patient level: action or retrospective introspection. Radiother Oncol 1992;25:146-7.

4. Hulshof M, Vanuytsel L, Van den Bogaert W, van der Schueren E. Localization errors in mantlefield irradiation for Hodgkin's disease. Int J Radiat Oncol Biol Phys 1989;17:679-83.

5. Lebesque JV, Bel A, Bijhold J. Detection of systematic patient setup errors by portal film analysis. Radiother Oncol 1992;23:198-203.

6. Leunens G, Verstraete J, Van den Bogaert W, Van Dam J, Dutreix A, van der Schueren E. Human errors in data transfer during the preparation and delivery of radiation treatment affecting the final result: "garbage in, garbage out". Radiother Oncol 1992;23:217-22.

7. Mitine C, Leunens G, Verstraete J, et al. Is it necessary to repeat quality control procedures for head and neck patients? Radiother Oncol 1991;21: 201-10.
8. Wallner PE, Lustig RA, Pajak TF, et al. Impact of initial quality control review on study outcome in lung and head/neck cancer studies - review of the Radiation Therapy Oncology Group experience. Int J Radiat Oncol Biol Phys 1989;17:893-900.

9. Calandrino R, Cattaneo GM, Del Vecchio A, Fiorino C, Longobardi B, Signorotto P. Human errors in the calculation of monitor units in clinical radiotherapy practice. Radiother Oncol 1993;28: 86-8.

10. Cohen L, Schultheiss TE, Kennaugh RC. A radiation overdose incident: initial data. Int J Radiat Oncol Biol Phys 1995;33:217-24.

11. Escó R, López P, Bellosta R, Baquedano JE, Mateo P. Accidental overirradiation syndrome. Radiother Oncol 1993;28:177-8.

12. Cohen L, Schultheiss T, Kennaugh RC. A radiation overdose incident: initial data. Int J Radiat Oncol Biol Phys 1995;33:217-24.

13. Woudstra E, Huizenga $H$, van de Poel JA. Possible leakage radiation during malfunctioning of a Sagittaire accelerator. Radiother Oncol 1993;29: 39-44.

14. Haywood J. Radiotherapy accidents in Europe - a preliminary report. Scope 1993;2:45-6. Citado por Duggan L, Kron T, Howlett S, Skov A, O'Brien P. An independent check of treatment plan, prescription and dose calculation as a QA procedure. Radiother Oncol 1997;42:297-301.

15. Mota HC, Almeida CE, Di Prinzio R. Report of an accidental exposure of patients in radiation therapy. Proceedings of the World Congress on Medical Physics and Biomedical Engineering, Nice, France, 1997.

16. Borras C, Marenco H, Bermudez L, Gimenez JC. Dosimetry report of a radiation overexposure of 109 radiotherapy patients. Proceedings of the World Congress on Medical Physics and Biomedical Engineering, Nice, France, 1997.
17. Kutcher GJ, Coia L, Gillin M, et al. Comprehensive QA for radiation oncology: report of AAPM Radiation Therapy Committee Task Group 40. Med Phys 1994;21:581-618.

18. World Health Organization. Quality assurance in radiotherapy. Génève: WHO, 1988.

19. Resolução da Secretaria Estadual da Saúde SS625,14 dezembro/1994. Uso, posse e armazenamento de fontes de radiação ionizante no âmbito do Estado de São Paulo.

20. Norma Experimental CNEN-NE-3.06. Requisitos de radioproteção e segurança para serviços de radioterapia. Diário Oficial da União, 30/3/1990.

21. Redmond PW. Nuclear regulatory commission report. Med Dosim 1988;13:87-93.

22. Hanks GE. Quality assurance in radiation oncology in the United States 1988. Cancer 1989;64(1 Suppl):223-5.

23. Masterson ME, Barest G, Chui CS, et al. Interinstitutional experience in verification of external photon dose calculations. Int J Radiat Oncol Biol Phys 1991;21:37-58.

24. Rozenfeld M, Jette D. Quality assurance of radiation dosage: usefulness of redundancy. Radiology 1984;150:241-4.

25. Duggan L, Kron T, Howlett S, Skov A, O'Brien P. An independent check of treatment plan, prescription and dose calculation as a QA procedure. Radiother Oncol 1997;42:297-301.

26. Calandrino R, Cattaneo GM, Fiorino C, Longobardi B, Mangili P, Signorotto P. Detection of systematic errors in external radiotherapy before treatment delivery. Radiother Oncol 1997;45: 271-4.

27. Barthelemy-Brichant N, Sabatier J, Dewé W, Albert A, Deneufbourg J-M. Evaluation of frequency and type of errors detected by a computerized record and verify system during radiation treatment. Radiother Oncol 1999;53:149-54. 